
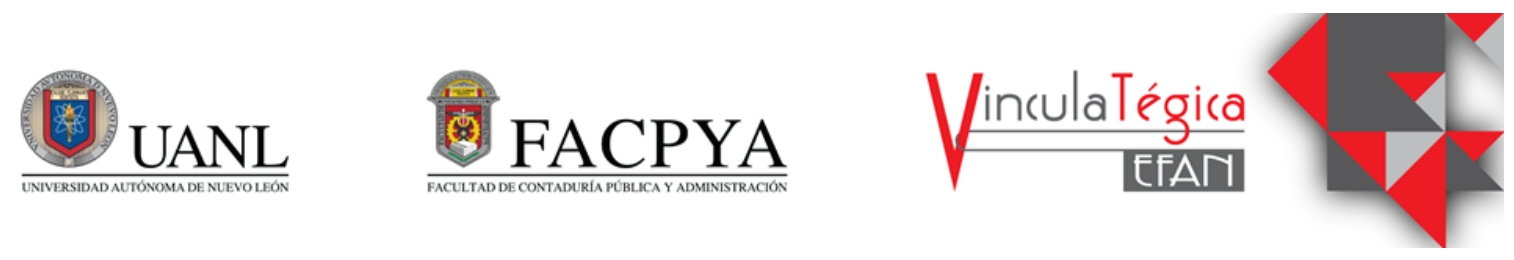

\title{
Compliance en el Sistema Nacional Anticorrupción de las Entidades Fiscalizadas de la Federación
}

\author{
Salvador Sánchez Ruanova ${ }^{1}$, Edgar Tapia Lara ${ }^{2}$ y Gerardo Hernández Barrena ${ }^{3}$ \\ ${ }^{1}$ Benemérita Universidad Autónoma de Puebla, salvador.sanchez@correo.buap.mx, Val Sequillo 70, \\ Universidades, 72000 Puebla, Pue., 2225667310. \\ ${ }^{2}$ Benemérita Universidad Autónoma de Puebla, edgar.tapia@correo.buap.mx, Val Sequillo 70, \\ Universidades, 72000 Puebla, Pue., 2222701919. \\ ${ }^{3}$ Benemérita Universidad Autónoma de Puebla, gerardo.hernandez@correo.buap.mx, Val Sequillo 70, \\ Universidades, 72000 Puebla, Pue., 2221354099.
}

\author{
Información del artículo revisado por pares \\ Fecha de aceptación: junio-2021 \\ Fecha de publicación en línea: diciembre-2021 \\ DOI: https://doi.org/10.29105/vtga7.1-148
}

\section{RESUMEN}

Lo relevante de esta investigación se enfoca principalmente en la verificación del cumplimiento del Sistema Nacional Anticorrupción de las facultades establecidas en la Ley General del Sistema Nacional Anticorrupción debido a la necesidad de mitigar la corrupción en los distintos organismos encargados de vigilar la correcta aplicación de los recursos públicos, ya que en México cada vez son más los casos de impunidad que sobresalen debido a la falta de ética y moral de los servidores públicos, es por ello que se pretende realizar una propuesta de establecer un "Compliance Administrativo" que sirva de apoyo a las Entidades Fiscalizadas establecidas en el país, así como a los organismos encargados de llevar a cabo la fiscalización y transparencia en México para enfocarse en prevenir y detectar, para sancionar las faltas administrativas o hechos de corrupción que puedan existir por parte de los servidores públicos al momento de realizar sus actividades dentro del cargo que les corresponde, tomando en cuenta siempre el marco legal vigente que le sea aplicable, con la intención de generar nuevamente la confianza de los ciudadanos en su gobierno, evitar la impunidad y lograr obtener una evaluación a tiempo real del manejo del erario público con base en el control interno, manuales de organización, códigos de ética y conducta, sistemas de denuncia y la capacitación continua.

Palabras Claves: Compliance, Control Interno, Anticorrupción.

Códigos JEL: H11 Estructura, alcance y desempeño del gobierno; H21 Eficiencia, Fiscalidad óptima; H83 Administración pública, Contabilidad y auditorías del sector público; M48 Política y regulación del gobierno.

\section{ABSTRACT}

What is relevant in this investigation is mainly focused on verifying compliance with the National Anticorruption System of the powers established in the General Law of the National Anticorruption System due to the need to mitigate corruption in the different agencies in charge of monitoring the correct application of resources. public, since in Mexico there are more and more cases of impunity that stand out due to the lack of ethics and morals of public servants, that is why it is intended to make a proposal to establish an "Administrative Compliance" that serves to support the Audited Entities established in the country, as well as the bodies in charge of carrying out the inspection and transparency in Mexico to focus on preventing and detecting, to punish administrative misconduct or acts of corruption that may exist on the part of public servants when time to carry out their activities within the position that corresponds to them, always taking into account the current legal 
framework that is applicable, with the intention of generating again the confidence of citizens in their government, avoiding impunity and obtaining a real-time evaluation of the management of the public treasury based on internal control , organization manuals, codes of ethics and conduct, complaint systems and ongoing training.

JEL Codes: H11 Structure, scope and performance of the government; H21 Efficiency, Optimal Taxation; H83 Public administration, Accounting and audits of the public sector; M48 Government policy and regulation.

\section{INTRODUCCIÓN}

En México han ocurrido sucesos históricos en los que el país se ha encontrado con distintas problemáticas de corrupción, fraudes, impunidad, enriquecimiento ilícito, peculado, etc., lo que es preocupante debido a que se crea un estancamiento económico, evitando que podamos alcanzar un desarrollo cada vez más alto como nación. Podrían existir muchos factores que hacen que la corrupción crezca preponderantemente, pero principalmente encontramos la falta de una administración sincronizada, que vigile las acciones dentro de las Entidades Fiscalizadas.

En la reforma publicada el día 27 de mayo del 2015 en el Diario Oficial de la Federación (DOF), al artículo 113 de la Constitución Política de los Estados Unidos Mexicanos (CPEUM) destaca la creación del Sistema Nacional Anticorrupción, (Secretaría de Gobernación, 2015). Con el fin de que esta sirva como instancia reguladora entre todas las autoridades de cada uno de los órdenes de gobierno para buscar y fomentar la rendición de cuentas, erradicar la corrupción y la impunidad. (Camára de Diputados del $\mathrm{H}$. Congreso de la Unión, 2021)

Derivado de lo anterior, se fija como objetivo del Sistema Nacional Anticorrupción (SNA) establecer mecanismos de coordinación entre la Federación, las Entidades federativas y los municipios, así como las bases mínimas para la prevención de hechos de corrupción y faltas administrativas y emitir políticas públicas que contemplen el combate a la corrupción, la fiscalización y el control de los recursos públicos.

Este Sistema Nacional Anticorrupción (SNA) se forma por la integración de un Comité Coordinador, el Comité de
Participación Ciudadana, el Comité Rector del Sistema Nacional de Fiscalización y los Sistemas Locales. (Cámara de Diputados del H. Congreso de la Unión, 2016)

El Comité Coordinador se integra por las siguientes figuras: un representante del Comité de Participación Ciudadana (CPC), la Auditoria Superior de la Federación (ASF), la Fiscalía Especializada de Combate a la Corrupción (FECC), la Secretaría de la Función Pública (SFP), el Consejo de la Judicatura Federal (CJF), el Instituto Nacional de Transparencia, Acceso a la Información y Protección de Datos Personales y el Tribunal Federal de Justicia Administrativa. (Cámara de Diputados del H. Congreso de la Unión, 2016)

El Comité de participación ciudadana se conforma por cinco ciudadanos que cuenten con la ética y moral impecable y que siempre estén interesados en contribuir a la transparencia, la rendición de cuentas y la erradicación de la corrupción.

El SNA y SNF están apostando en la evolución de la inteligencia artificial para combatir la corrupción mediante las Tecnologías de la Información y Comunicación por una Plataforma Digital Nacional (PDN) que actúe como un detector de posibles faltas administrativas, para así lograr la aplicación de sanciones, realizar una fiscalización eficiente de los recursos públicos, y a su vez lograr que la información sea compartida de manera más sencilla, que cualquier persona o institución interesada pueda tener acceso con tan solo conectarse a una red, además de instrumentar políticas integrales que les permitan lograr un desarrollo optimo en el desempeño de sus funciones.

Actualmente en la normatividad mexicana se busca la prevención de la corrupción y sobre la inclusión de las políticas integrales aplicables al servicio públicoprivado que fortalezcan la ética de los funcionarios y particulares, conductas que se rigen sobre un estado de derecho. Sin embargo, este estado se corrompe con mayor facilidad y sobresale de los beneficios que se adquieren por derecho, por lo que es necesario la aplicación del marco legal que tiene como 
objetivo efectuar la imposición de sanciones y regule dichos comportamientos.

Las Entidades Públicas de nuestro país carecen de políticas integrales que les permitan la mejora continua y un correcto desempeño de sus funciones en correlación con la aplicación de los ingresos captados, desafortunadamente hasta la fecha no se ha diseñado una metodología para el sector público instrumentada en un decálogo de valores que trae consigo como consecuencia las faltas administrativas y hechos de corrupción en los que están inmiscuidos servidores públicos y privados. Es por esto por lo que se indagará sobre el cumplimiento del marco normativo aplicable al SNA, tomando como base las facultades de cada uno de sus integrantes, en especial del Comité Coordinador, que es quien se encarga de que todas las actividades se realicen en armonía.

En esta investigación se planteará una propuesta de "Compliance Administrativo" que sea una guía eficiente en el desempeño de las administraciones y permita guiar a los servidores públicos para lograr los objetivos establecidos conforme a su papel fundamental en la realización de sus actividades asignadas, tomando en cuenta los manuales, códigos de conducta, políticas y sanciones correspondientes, entre otros puntos relevantes.

\section{MARCO TEÓRICO}

El año 2015, para nuestro país fue un parteaguas gracias a reforma de la Constitución Política de los Estados Unidos Mexicanos (CPEUM) en su "Título IV "De las Responsabilidades de los Servidores Públicos, Particulares Vinculados con Faltas Administrativas Graves o Hechos de Corrupción, y Patrimonial del Estado" Públicos, Particulares Vinculados con Faltas Administrativas Graves o Hechos de Corrupción, y Patrimonial del Estado" (Secretaría de Gobernación, 2015), misma en la que se señaló en la exposición de motivos los altos índices de corrupción que existen en los diferentes órdenes de gobierno del Estado Mexicano y por tanto era de vital importancia reformar nuestras normas jurídicas $y$ emprender al cambio de los principios éticos y cívicos de los servidores públicos y la ciudadanía.

"El 7 y 15 de mayo se reformaron los artículos 22, 28, 41, 73, 74, 76, 104, 109, 110, 113, 114, 116 y 122 de la Constitución Política de los Estados Unidos Mexicanos, con el fin de combatir y erradicar la corrupción e impulsar un cambio en la evaluación, fiscalización y desempeño de las políticas públicas en nuestro país. Se busca el establecimiento de una Plataforma Digital Nacional (PDN) que permita optimizar las auditorías del gasto público, mejorar la calidad y transparencia en la administración del erario en los tres órdenes de gobierno, a través de la medición de la eficiencia, eficacia, economía, transparencia y honradez, dando origen a una nueva etapa en la fiscalización y cumplimiento en nuestro país". (Secretaría de Gobernación, 2015)

Las reformas a la CPEUM dieron origen a nuevos ordenamientos legales como la Ley General del Sistema Nacional Anticorrupción (LGSNA) publicada el 18 de Julio del 2016 en el Diario Oficial de la Federación (DOF), misma que en su artículo 6 establece que el Sistema Nacional Anticorrupción (SNA) es el encargado de establecer los principios, bases generales, políticas públicas y procedimientos para la correcta coordinación entre las autoridades de todos los órdenes de gobierno con el objetivo de prevenir, detectar y sancionar las faltas administrativas y hechos de corrupción incurridos por servidores públicos o particulares y evaluar las políticas públicas. (Camara de Diputados del $\mathrm{H}$. Congreso de la Unión, 2016)

El gobierno mexicano está diseñando un sistema que permita fortalecer las políticas anticorrupción mediante la creación del SNA, apoyado recíprocamente con el Sistema Nacional de Fiscalización, busca que las Entidades de Fiscalización Superior (EFS) presenten sus informes en la plataforma digital en apego a seis principios clave que permitirán la aplicación de responsabilidades en el menos tiempo posible sin tener que esperar por lapsos mayores a un año, al estar integrando los órganos de auditoría y control gubernamental de los diferentes órdenes de gobierno y mediante la plataforma para coordinarse, intercambiar información e impulsar mejoras 
prácticas en las políticas de control interno y fiscalización.

Además otros ordenamientos legales que se reformaron en el Congreso de la Unión para cimentar las políticas públicas que coadyuben el combate a la corrupción son las siguientes: Ley de Coordinación Fiscal, Código Penal Federal, Ley Orgánica de la Procuraduría General de la República, Ley General de Contabilidad Gubernamental, Ley Orgánica de la Administración Pública Federal y se crearon nuevas: la Ley Orgánica del Tribunal Federal de Justicia Administrativa, Ley General de Responsabilidades Administrativas y la Ley de Fiscalización y Rendición de Cuentas de la Federación, todas publicadas en el Diario Oficial de la Federación (DOF) del 18 de Julio de 2016 y cada una de ellas para fortalecer el SNA. (Camara de Diputados del H. Congreso de la Unión, 2016)

El SNA es el encargado de realizar y publicar las políticas integrales que ayuden a una prevención adecuada y precisa que ayude a obtener el control de las faltas administrativas que llegarán a manifestarse dentro de una Entidad, a su vez se buscar realizar la fiscalización y el control de recursos públicos de manera abierta y accesible a cualquier persona, diseñando los parámetros que sirven para medir y dar seguimiento a los informes de las evaluaciones de los avances y resultados del ejercicio de las funciones y de la aplicación de las políticas y programas desempeño y funcionamiento del SNA.

Deberá ser participe en el desarrollo y aplicación de las bases y principios que fomentan una buena coordinación de las autoridades de cada uno de los órdenes de gobierno en cuanto a la fiscalización, además de rendir recomendaciones las cuales no tiene la obligación se realizar, sin embargo, estas podrán ser dirigidas a las autoridades que se involucren, según el informe anual y dar seguimiento a los resultados sistematizados por parte de las autoridades.

El Sistema Nacional de Fiscalización (SNF) estará conformado por: "un comité rector y cinco grupos de trabajo que actuaran de manera conjunta y simultánea, mismos que serán los responsables de establecer el marco jurídico y metodológico que regirá la fiscalización, y a su vez, verificar que se dé cumplimiento a la Ley General de Contabilidad Gubernamental, evaluar la armonización contable y crear el diseño de un sistema de denuncias que sirva para aumentar la participación ciudadana." (Cámara de Diputados del H. Congreso de la Unión, 2016) Con lo anteriormente expuesto se contempla que, para llevar a cabo una fiscalización de manera óptima y fiable, se requieren de políticas que ayuden al mejor manejo y difusión de la información pública, sin embargo, no se ha implementado el cumplimiento de las Entidades Fiscalizadoras para llevar a cabo la publicación en la Plataforma Digital Nacional.

\section{MÉTODO}

\section{Objetivos:}

A) Generales. Verificar el cumplimiento por parte del Sistema Nacional Anticorrupción en cuanto a las disposiciones normativas que le competen y si cuenta con una política de integridad y lograr así, optar por la implementación de un Compliance Administrativo que apoye a la ejecución de las facultades del SNA.

B) Específicos. Lograr la integración de un Compliance Administrativo en las Entidades Fiscalizadas a nivel federal que rinden cuentas a la nación con el fin de coadyuvar al Sistema Nacional Anticorrupción a combatir la corrupción y prevenir que los servidores públicos recaigan en faltas administrativas con base en la Ley General de Responsabilidades Administrativas.

2. Planteamiento del problema: el problema con el que nos enfrentamos al momento de realizar esta investigación recae en el incumplimiento, por parte de la mayoría de las Entidades Fiscalizadas de México, a la política de integridad generando rezagos en la difusión de su información y transparencia, por lo que al no contar con información a evaluar resulta imposible detectar posibles acciones de corrupción y el Sistema Nacional Anticorrupción no puede realizar su atribución de coordinación entre las autoridades correspondientes.

2. Diseño: El método mixto (Deductivo- 
Inductivo) será en el que nos basaremos para que se realice esta investigación, debido a que los aspectos generales se simplificarán y tratarán de llevar a cabo en aspectos específicos con el fin de deliberar un dato exacto.

2. Población: La población con la que se realizó la investigación recae en el Sistema Nacional Anticorrupción, así como en las organizaciones encargadas de la fiscalización y supervisión del cumplimiento de las disposiciones normativas, por otra parte, podrá aplicarse a cualquier entidad del sector Público o Privado de la nación mexicana.

3. Entorno: El entorno del proyecto radica en la supervisión constante de la Plataforma del Sistema Nacional Anticorrupción para profundizar en el cumplimiento de las disposiciones normativas que regulan al mismo.

4. Intervenciones: Las leyes y las demás plataformas digitales oficiales de las dependencias de gobierno serán nuestra primordial fuente de investigación para analizar los temas detallados verificando artículos y apartados relacionados con el tema.

5. Análisis estadístico: Se realizó la recolección y observación de los distintos informes, manuales, políticas y lineamientos emitidos por el Comité Coordinador, comparando con lo establecido en la Ley General del Sistema Nacional Anticorrupción, y así lograr verificar que se cuente con cada una de las facultades atribuidas a este.

\section{RESULTADOS}

Se verificó el cumplimiento de cada una de las facultades especificadas en el artículo 9 de la Ley General del Sistema Nacional Anticorrupción (Cámara de Diputados del H. Congreso de la Unión, 2016) y después de analizar la página de internet oficial del Sistema Nacional Anticorrupción (SNA) encontramos que no se cumple con la totalidad de lo establecido legalmente, en algunas facultades más relevantes se observó lo siguiente:

$>$ Elaborar un programa de trabajo anual.

Para esta facultad nos percatamos de que se requiere un programa de trabajo anual con el fin de planificar y gestionar los fines del
SNA, es por ello por lo que se muestran las políticas nacionales, recomendaciones, vinculación, incorporación de la participación ciudadana, herramientas, etc., y el avance de dicho programa se reportara en las sesiones que se celebren.

Establecer bases y principios que apoyen a la coordinación de sus integrantes.

Para llevar a cabo el trabajo en armonía de los integrantes del SNA es necesario que existan las bases y principios que fomenten la buena interacción, comunicación e intercambio de la información entre ellos.

Diseñar y promocionar la política nacional, realizar evaluaciones periódicas, los ajustes y realización de las modificaciones correspondientes.

La política nacional anticorrupción se enfoca en involucrar 4 ejes principales; "1) Combatir la corrupción y la impunidad, 2) Combatir la arbitrariedad y el abuso de poder, 3) Promover la mejora de la gestión pública y de los puntos de contacto gobierno - sociedad, 4) Involucrar a la sociedad y el sector privado" (Comité Coordinador del SNA, 2020). En cada uno de ellos se enlistan las prioridades más relevantes para cumplir con su objetivo particular, tales como "Fortalecer las capacidades de investigación de la Fiscalía General de la República y de las fiscalías de las entidades federativas en materia de delitos por hechos de corrupción, Implementar un modelo de evaluación del desempeño del Sistema Nacional Anticorrupción, Desarrollar e implementar un sistema de información que contenga las compras y adquisiciones que hacen la entidades públicas, Crear un catálogo nacional de mecanismos de participación social que contribuya a la incidencia formal y efectiva de la vigilancia ciudadana en la prevención y combate de la corrupción, entre otras" (Secretaría Ejecutiva SNA, 2020).

Con base en el resultado de las evaluaciones que realice la Secretaría Ejecutiva se acordarán las medidas o las modificaciones correspondientes a las políticas integrales y se requerirá la información correspondiente a las Entidades públicas referentes al cumplimiento de la política nacional y las políticas integrales implementadas. 
Esta fracción se encuentra en proceso de cumplimiento debido a que la implementación de los sistemas locales en las 32 entidades federativas en 13 ha sido aprobada, 7 se encuentra atendiendo la retroalimentación, 10 en proceso de elaboración y 2 no han iniciado su proceso de elaboración. (Secretaría Ejecutiva del SNA, 2021)

Emitir un informe anual que sobre los avances y resultados obtenidos en el ejercicio de sus funciones y de la aplicación de políticas y programas.

El Comité Coordinador del SNA ha cumplido con la presentación y publicación de su informe anual de actividades y de resultados 2020 en su sesión Ordinaria realizada en el año 2021, en el cual se expresa la aplicación de los recursos financieros, humanos, las investigaciones realizadas en materia de denuncias interpuestas, el seguimiento realizado al informe anterior y las propuestas adicionales recomendadas en el aspecto de transparencia y la participación de la ciudadanía como víctimas en delitos de corrupción.

$>$ Emitir recomendaciones públicas no vinculantes ante las autoridades vigilando el seguimiento constante.

El Comité Coordinador ha realizado su "Quinto Informe de Seguimiento a la recomendación no vinculante dirigida a los Poderes Ejecutivo y Legislativo de las Entidades Federativas relacionada con la selección de Jueces y Magistrados" correspondiente al ejercicio 2020, por lo que se ha cumplido con la facultad correspondiente.

Establecer una Plataforma Digital que integre y conecte los sistemas electrónicos que posean datos e información necesaria para que las autoridades competentes tengan acceso a la evolución patrimonial, de declaración de intereses y constancia de presentación de declaración fiscal de los Servidores públicos, así como aquellos que intervengan en procedimientos de contrataciones públicas, los que se encuentren sancionados, el proceso de Fiscalización realizado, un sistema de denuncias y la información referente a las contrataciones que se realicen en las entidades en todos los niveles de gobierno.
Se ha creado la Plataforma Digital Nacional, la cual cumple con lo establecido en el artículo 49 de la Ley General del Sistema Nacional Anticorrupción, que establece los sistemas electrónicos mínimos con los que debe contar dicha plataforma: "Sistema de evolución patrimonial, de declaración de intereses y constancia de presentación de declaración fiscal, Sistema de los Servidores públicos que intervengan en procedimientos de licitaciones públicas, Sistema nacional de Servidores públicos y particulares sancionados, Sistema de información y comunicación del Sistema Nacional y del Sistema Nacional de Fiscalización, Sistema de denuncias públicas por faltas administrativas y hechos de corrupción y el Sistema de Información Pública de Contrataciones." (Cámara de Diputados del H. Congreso de la Unión, 2016)

$>\quad$ Lograr la celebración de convenios de coordinación, colaboración y concertación para que se realice el cumplimiento de los objetivos del Sistema Nacional

Se ha llevado a cabo la tarea de celebrar nuevos convenios de colaboración con distintas entidades, entre ellos la Fiscalía General de la Republica, el Instituto Nacional de Transparencia, Acceso a la Información y Protección de Datos Personales, el Órgano Interno del Control del INE, Secretaria de la Función Pública la Unidad de Inteligencia Financiera de la SHCP, entre otras.

\section{PROPUESTA}

Como podemos observar el Sistema Nacional Anticorrupción va cumpliendo en mayor parte con las competencias que le corresponden, sin embargo, existe la posibilidad de mejorarlo y hacer que se obtenga un cumplimiento total partiendo de la instrumentación de un Compliance Administrativo en todas y cada una de las Entidades Fiscalizadas que rinden cuentas a la nación, debido al fundamento que se está investigando a nivel federal ya que está diseñado con base en el artículo 25 de la Ley General de Responsabilidades Administrativas que nos indica los elementos fundamentales para contar con una política de integridad, tales como manuales, código de ética, conducta y un sistema de integridad, por 
lo que se puede adecuar a cualquier entidad pública para que sirva de guía para el cumplimiento del sector público, adicionando los elementos básicos para conocer a una dependencia pública sus objetivos y fines establecidos, siendo nuestra propuesta la siguiente:

\section{COMPLIANCE ADMINISTRATIVO}

1. Misión

2. Visión

3. Decálogo de Valores

4. Políticas Administrativas

5. Objetivos específicos

6. Manual de Organización

7. Manual de procedimientos por área administrativa

8. Manual de calidad

9. Manual de recursos humanos

10. Manual de transparencia

11. Organigrama general

12. Organigrama por área administrativa

13. Código de Ética y Conducta

14. Estudio de Evaluación al control Interno

15. Mapa de Riesgos

16. Manual de canales de comunicación

17. Manual de auditoría interna

18. Creación de los sistemas de control y vigilancia de los procesos administrativos

19. Sistema de denuncias

20. Sistema de quejas y sugerencias

21. Creación del Órgano Interno de Control

22. Lineamientos que rijan el actuar del Órgano Interno de Control

23. Sanciones

24. Sistema de entrenamiento y capacitación

25. Página de Internet o canal de radio que difunda las acciones realizadas

26. Informes mensuales en materia de:

a) Cumplimiento a las actividades

b) Desempeño de las funciones

c) Transparencia en rendición de cuentas

d) Servidores públicos sancionados

27. Informe de áreas de oportunidad

Comparando con los requisitos mínimos de un compliance, según los creadores de la revista española de la asociación ESCURA (Carbayo, 2020), encontramos que mencionan los siguientes:

1. Identificar las actividades propensas a fraudes para que puedan ser prevenidos. - En nuestro Compliance se considera un Manual de procedimientos por área administrativa, un Estudio de Evaluación al control Interno del cual derivado de este de crea un Mapa de Riesgos, por lo que se cumple con el primer requisito.

2. Establecer los protocolos o procedimientos que concreten el proceso de formación, adopción y ejecución de decisiones. - Se establecen diversos manuales y políticas administrativas que se harán cargo del buen manejo y realización de actividades siempre apegadas a un Código de Ética y Conducta.

3. Dispondrán de modelos de gestión de los recursos financieros adecuados para impedir la comisión de los delitos que deben ser prevenidos. - Para ello existe la Creación de los sistemas de control y vigilancia de los procesos administrativos que vigila el cumplimiento de las distintas áreas de la Entidad, incluida el área encargada de los recursos financieros.

4. Impondrán la obligación de informar de posibles riesgos e incumplimientos al organismo encargado de vigilar el funcionamiento y observancia del modelo de prevención. - Se cuenta con la elaboración de informes mensuales que contienen la información referente al Cumplimiento a las actividades, Desempeño de las funciones, Transparencia en rendición de cuentas y Servidores públicos sancionados.

5. Establecerán un sistema disciplinario que sancione adecuadamente el incumplimiento de las medidas que establezca el modelo. - Se cuenta con un apartado de Sanciones y los Sistemas correspondientes.

Con este compliance administrativo se busca cambiar el paradigma de los organismos que integran y rinden cuentas al Sistema Nacional Anticorrupción, con el fin de cumplir con los objetivos de este, es muy importante recordar que la corrupción siempre ha sido un tema de suma importancia y preocupación, con esto se logra una mejora para el avance que se necesita en este combate.

Se tiene que tomar en cuenta los principios de gobernanza que ayudan a las políticas anticorrupción ya que, al enfocarse en combatir los actos de irresponsabilidad o fraudes de los servidores públicos, es 
necesario contemplar los siguientes: 1) Estado de derecho, que significa que la ley está por encima de todos y se aplica de manera justa; 2) Transparencia, todos los ciudadanos tienen derecho a la información pública; 3) Rendición de cuentas, es la obligación de los servidores públicos hacia la ciudadanía para evaluar el actuar de los responsables; 4) Ética y Probidad, implica que se debe actuar con honestidad e integridad. (Miguel, 2021)

Al instrumentar una política integral para el cumplimiento de los objetivos de los organismos nos dará claridad en la realización de los procedimiento y actividades que realizan, de ahí la importancia y trascendencia de que cuenten con sus manuales, códigos, reglamentos, sistemas de comunicación y páginas de internet que les permitan desarrollar sus actividades de forma oportuna, veraz, eficiente y que a su vez den seguridad de sus actos ante la ciudadanía, mediante los portales de transparencia que ha implementado el gobierno.

\section{CONCLUSIONES}

Derivado de la investigación, nos percatamos que aún es necesario el cumplimiento de algunas disposiciones normativas o en su caso seguimiento y actualización de estas toda vez que se manejan datos ambiguos correspondientes a ejercicios anteriores, tal es el caso de la Plataforma Digital Nacional, toda vez que se encuentra implementada pero no se ha cumplido con la publicación en cada uno de los Estados de México, por ejemplo, en el caso de Puebla no se ha publicado el apartado de evolución patrimonial, declaración de intereses y declaración fiscal de los servidores públicos que operan en la Entidad, como este caso hay muchas más entidades que no han cumplido con esta aplicación (Secretaría Ejecutiva del SNA, 2021). En cuanto a la información recabada no se puede considerar de fácil acceso a cualquier persona que desea consultar la página oficial del Sistema Nacional Anticorrupción, pues como tal no existe un apartado único en donde se almacene toda la información referente a informes, manuales, políticas, convenios de colaboración, acuerdos, recomendaciones y demás documentación elaborada por los integrantes del SNA.

Es por ello por lo que se realiza la propuesta de un compliace administrativo que ayudará a los integrantes del SNA a llevar un mejor control y manejo de estos documentos, siendo adaptable a cualquier entidad pública, con la visión de que se alcance cada vez más la transparencia, se prevengan los actos de corrupción y se ejerza justicia en aquellos servidores públicos que han realizado faltas administrativas sin la oportunidad de salir impunes ante sus acciones. 


\section{REFERENCIAS}

Cámara de Diputados del H. Congreso de la Unión. (18 de 07 de 2016). Ley General del Sistema Nacional Anticorrupción. http://www.diputados.gob.mx/LeyesBiblio/pdf/LGSNA.pdf

Camára de Diputados del H. Congreso de la Unión. (11 de 03 de 2021). Constitución Política de los Estados Unidos Mexicanos. Obtenido de http://www.diputados.gob.mx/LeyesBiblio/pdf/1_110321.pdf

Carbayo, V. J. (2020). Los requisitos mínimos de un programa de Compliance. ESCURA, 11. Obtenido de https://www.escura.com/archivos/pdf/Revista-compliance-ES-web.pdf

Comité Coordinador del SNA. (2020). Política Nacional Anticorrupción. México: Secretaría Ejecutiva SNA.

Miguel, C. R. (20 de 05 de 2021). Requena Abogados. Obtenido de El Compliance en el Sector Público Mexicano: https://requena.org.mx/wpcontent/uploads/2019/11/COMPLIANCE_SectorPublico_vF.pdf

Secretaría de Gobernación. (27 de 05 de 2015). Diario Oficial de la Federación. Recuperado el 15 de $05 \quad$ de $2020, \quad$ de

http://dof.gob.mx/nota_detalle.php?codigo $=5394003 \&$ fecha $=27 / 05 / 2015$

Secretaría Ejecutiva del SNA. (10 de 05 de 2021). Plataforma Digital Nacional. Obtenido de https://www.plataformadigitalnacional.org/about

Secretaría Ejecutiva del SNA. (16 de 04 de 2021). Sistema Nacional Anticorrupción. Obtenido de POLÍTICAS ESTATALES: https://sna.org.mx/politicas-estatales-anticorrupcion/

Secretaría Ejecutiva SNA. (29 de 01 de 2020). Política Nacional Anticorrupción. Obtenido de PNA (VERSIÓN COMPLETA): $\quad$ https://www.sesna.gob.mx/wpcontent/uploads/2020/02/Pol\%C3\%ADtica-Nacional-Anticorrupci\%C3\%B3n.pdf 